$\operatorname{Conf}-941144--153$

LBL-36909

UC-404

$\mathbf{B}$

Lawrence Berkeley Laboratory

UNIVERSITY OF CALIFORNIA

Materials Sciences Division

National Center for Electron Microscopy

Presented at the Materials Research Society Symposium, Boston, MA, November 28-December 2, 1994, and to be published in the Proceedings

Size, Shape and Crystallinity of Luminescent Structures in Oxidized Si Nanoclusters and H-Passivated Porous Si

S. Schuppler, S.L. Friedman, M.A. Marcus, D.L. Adler, Y.-H. Xie, F.M. Ross, T.D. Harris, W.L. Brown, Y.J. Chabal, P.J. Szajowski, E.E. Chaban, L.E. Brus, and P.H. Citrin

November 1994

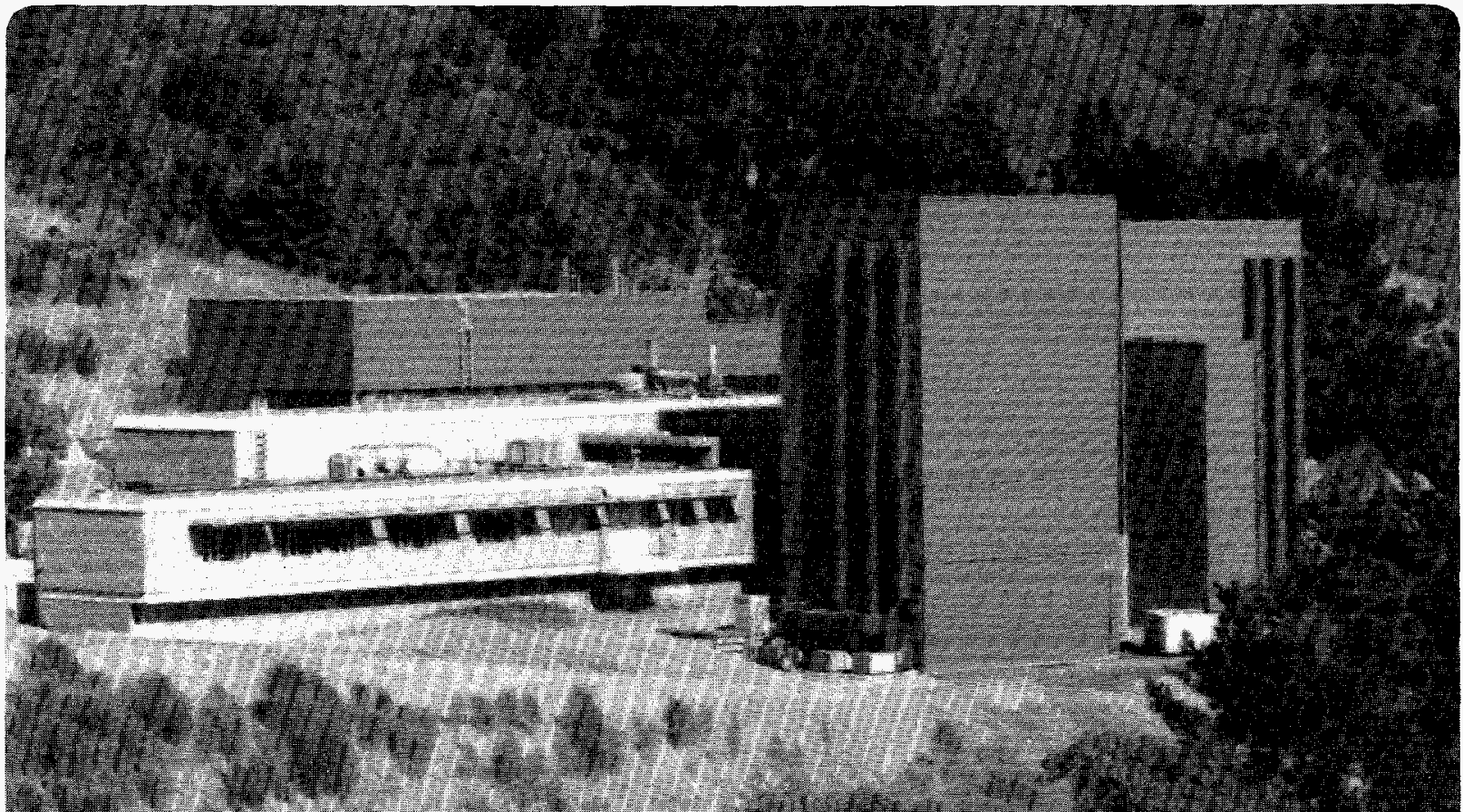




\section{DISCLAIMER}

This document was prepared as an account of work sponsored by the United States Government. Neither the United States Government nor any agency thereof, nor The Regents of the University of California, nor any of their employees, makes any warranty, express or implied, or assumes any legal liability or responsibility for the accuracy, completeness, or usefulness of any information, apparatus, product, or process disclosed, or represents that its use would not infringe privately owned rights. Reference herein to any specific commercial product, process, or service by its trade name, trademark, manufacturer, or otherwise, does not necessarily constitute or imply its endorsement, recommendation, or favoring by the United States Government or any agency thereof, or The Regents of the University of California. The views and opinions of authors expressed herein do not necessarily state or reflect those of the United. States Government or any agency thereof or The Regents of the University of California and shall not be used for advertising or product endorsement purposes.

Lawrence Berkeley Laboratory is an equal opportunity employer. 


\section{DISCLAIMER}

Portions of this document may be illegible in electronic image products. Images are produced from the best available original document. 
LBL-36909

\title{
Size, Shape and Crystallinity of Luminescent Structures in Oxidized Si Nanoclusters and H-Passivated Porous Si
}

S. Schuppler, S.L. Friedman*, M.A. Marcus, D.L. Adler\#, Y.-H. Xie, F.M. Ross†, T.D. Harris, W.L. Brown, Y.J. Chabal, P.J. Szajowski, E.E. Chaban, L.E. Brus and P.H. Citrin

\author{
AT\&T Bell Laboratories \\ Murray Hill, NJ 07974 \\ †Lawrence Berkeley Laboratory \\ Materials Sciences Division \\ National Center for Electron Microscopy \\ Berkeley, CA 94720 \\ *Department of Applied Physics \\ Stanford University \\ Palo Alto, CA 90210 \\ \#Department of Materials Science \\ University of Illinois \\ Urbana-Champaign, IL 61801
}

Proc. MRS, Vol 358, (1995).

This work was supported by the Director, Office of Energy Research, Office of Basic Energy Sciences, Materials Science Division of the U.S. Department of Energy under Contract No. DE-AC03-76SF00098, financial support from the Alexander-von-Humboldt Foundation. 


\title{
SIZE, SHAPE, AND CRYSTALLINITY OF LUMINESCENT STRUCTURES IN OXIDIZED Si NANOCLUSTERS AND H-PASSIVATED POROUS Si
}

\author{
S. SCHUPPLER, S. L. FRIEDMAN, ${ }^{\text {a) }}$ M. A. MARCUS, D. L. ADLER, ${ }^{\text {b) }}$ Y.-H. XIE, F. M. \\ ROSS, ${ }^{\dagger}$ T. D. HARRIS, W. L. BROWN, Y. J. CHABAL, P. J. SZAJOWSKI, E. E. CHABAN, \\ L. E. BRUS, AND P. H. CITRIN
}

AT\&T Bell Laboratories, Murray Hill, NJ 07974

'National Center for Electron Microscopy, Lawrence Berkeley Laboratory, Berkeley, CA 94720

\begin{abstract}
Near-edge and extended $x$-ray absorption fine structure measurements from a wide variety of $\mathrm{H}$-passivated porous Si samples and oxidized Si nanocrystals, combined with electron microscopy, ir-absorption, $\alpha$-recoil, and luminescence emission data, provide a consistent structural picture of the species responsible for the luminescence observed in these systems. For luminescent porous Si samples peaking in the visible region, i. e., $\leq 700 \mathrm{~nm}$, their mass-weighted-average structures are determined here to be particles - not wires, whose short-range character is crystalline - not amorphous, and whose dimensions - typically $<15 \AA$ - are significantly smaller than previously reported or proposed. These results depend only on sample luminescence behavior, not on sample preparation details, and thus have general implications in describing the mechanism responsible for visible luminescence in porous silicon. New results are also presented which demonstrate that the observed luminescence is unrelated to either the photo-oxidized Si species in porous $\mathrm{Si}$ or the interfacial suboxide species in the Si nanocrystals.
\end{abstract}

\section{INTRODUCTION}

There has been intense interest in the visible room-temperature luminescence observed from anodically-grown porous silicon (por-Si) [1], from which a growing consensus has emerged to explain the luminescence with quantum-confined structures $[2,3]$. There remains, however, a basic lack of knowledge regarding the dimensions, shape, and crystallinity of the species actually responsible for the optical activity. This work summarizes recent $x$-ray absorption measurements [4] from a series of oxidized $\mathrm{Si}$ nanocrystals, whose shapes and sizes are known, and from a variety of anodically-grown (H-passivated) por-Si samples. The data, combined with luminescence emission measurements from each of the systems, establish new and unexpectedly smaller values for the average size of the luminescing Si structures. In addition, local crystallinity is observed and the importance of extended wire shapes is ruled out. New x-ray absorption, luminescence, and ir absorption data show that the observed luminescence in either the nanocrystals or the por-Si samples is not correlated with an oxygen-containing species. Our combined results have important implications for describing the origin of visible photoluminescence from por-Si. 


\section{EXPERIMENT}

The Si K-edge absorption measurements were performed at the National Synchrotron Light Source using the AT\&T X15B beamline [5]. A variety of por-Si samples prepared under very different conditions $[6,2,7,8]$ were studied with TEM, $\mathbf{x}$-ray- and irabsorption, $\alpha$-recoil, and luminescence excitation and emission spectroscopies. In order to minimize $O$ contamination, which was very easily detectable, air exposure of the freshly prepared samples was limited to $<10 \mathrm{~min}$ in all but the TEM and photo-oxidation measurements. These latter experiments involved collecting photoluminescence data as a function of laser exposure from various por-Si wafers followed by ir absorption from the differently irradiated and non-irradiated regions. Samples of the O-passivated (airinsensitive) Si nanocrystals [9] were prepared for x-ray absorption measurements by transferring them as colloidal suspensions onto graphite substrates.

\section{RESULTS}

\section{NEXAFS from Nanocrystals}

In Fig. 1(a) we show Si Knear-edge x-ray absorption fine structure (NEXAFS) data from three different sizes of oxidized Si nanocrystals, ox-Si ${ }_{x}$ labelled small $(s)$, medium $(m)$, and large ( $)$. A fourth, very small ( $v s$ ) sample was also measured but omitted in Fig. 1 (a) for clarity. Comparison with data from $c$ $\mathrm{Si}$ (freshly HF-rinsed to remove the native oxide film) and bulk $\mathrm{SiO}_{2}$ readily confirms that these are the two main components in the clusters [9]. A third, minor interfacial suboxide species, $\mathrm{SiO}_{x}$ is also observed and further discussed below. The $c-\mathrm{Si}, \mathrm{SiO}_{2}$, and ox- $\mathrm{Si}_{x}$ data have been normalized to common "edge jump" values, measured $>100 \mathrm{eV}$ above the Si K-edge where their $\mathrm{x}$-ray absorption intensities are structureless, i. e., atomiclike. The relative concentrations of $\mathrm{Si}$ and $\mathrm{SiO}_{2}$ in the nanocrystals (after density corrections) are directly proportional to their individual, easilyseparable edge jumps [10]. Now, $x$-ray diffraction, liquid chromatography, and TEM data [9] show that the $l, m, s$, and vs samples are $\mathrm{SiO}_{2}$-coated spheres of $\mathrm{Si}$ whose outer

Fig. 1. (a) Si K-edge NEXAFS data from $c$-Si, $\mathrm{SiO}_{2}$, and oxidized $\mathrm{Si}$ nanocrystals of different size, labelled small $(s)$, medium $(m)$, and large ( $)$. (b) NEXAFS data from $c$-Si, a$\mathrm{Si}$, and two differently prepared por-Si samples. All data in (a) and (b) are normalized to unity edge jump.

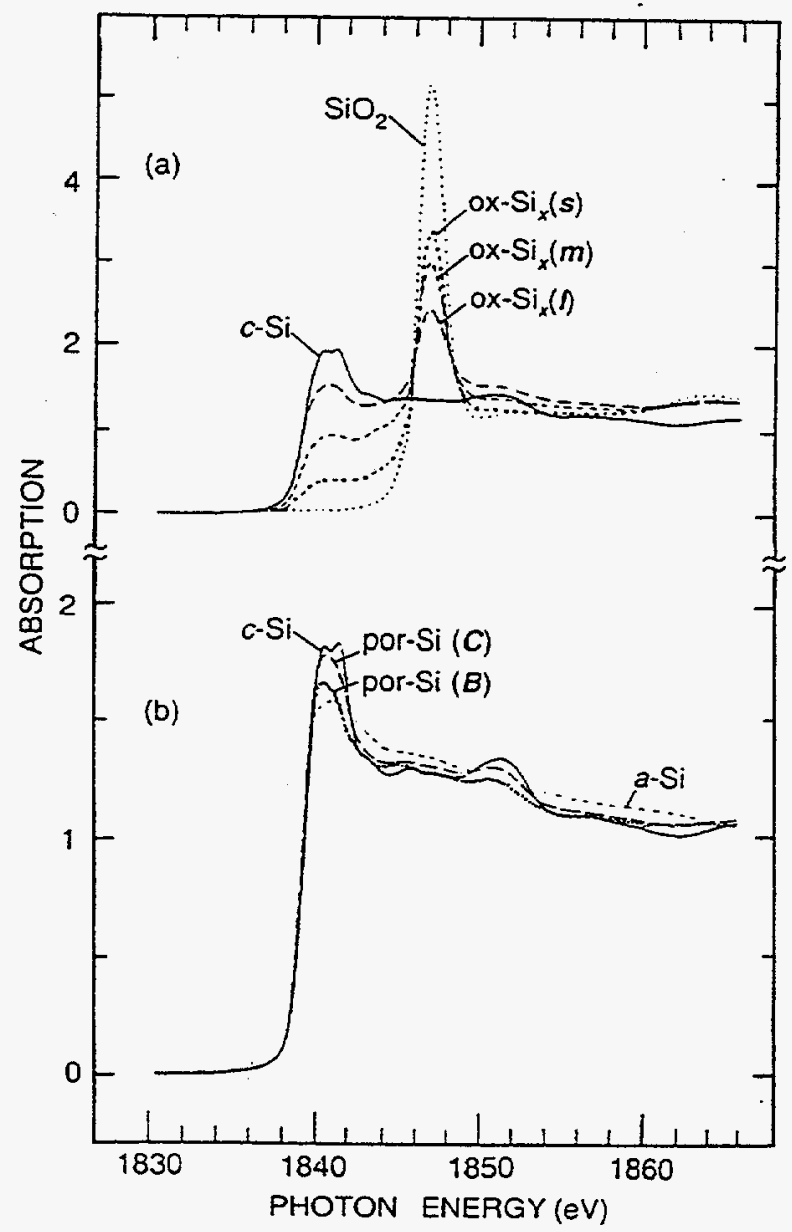




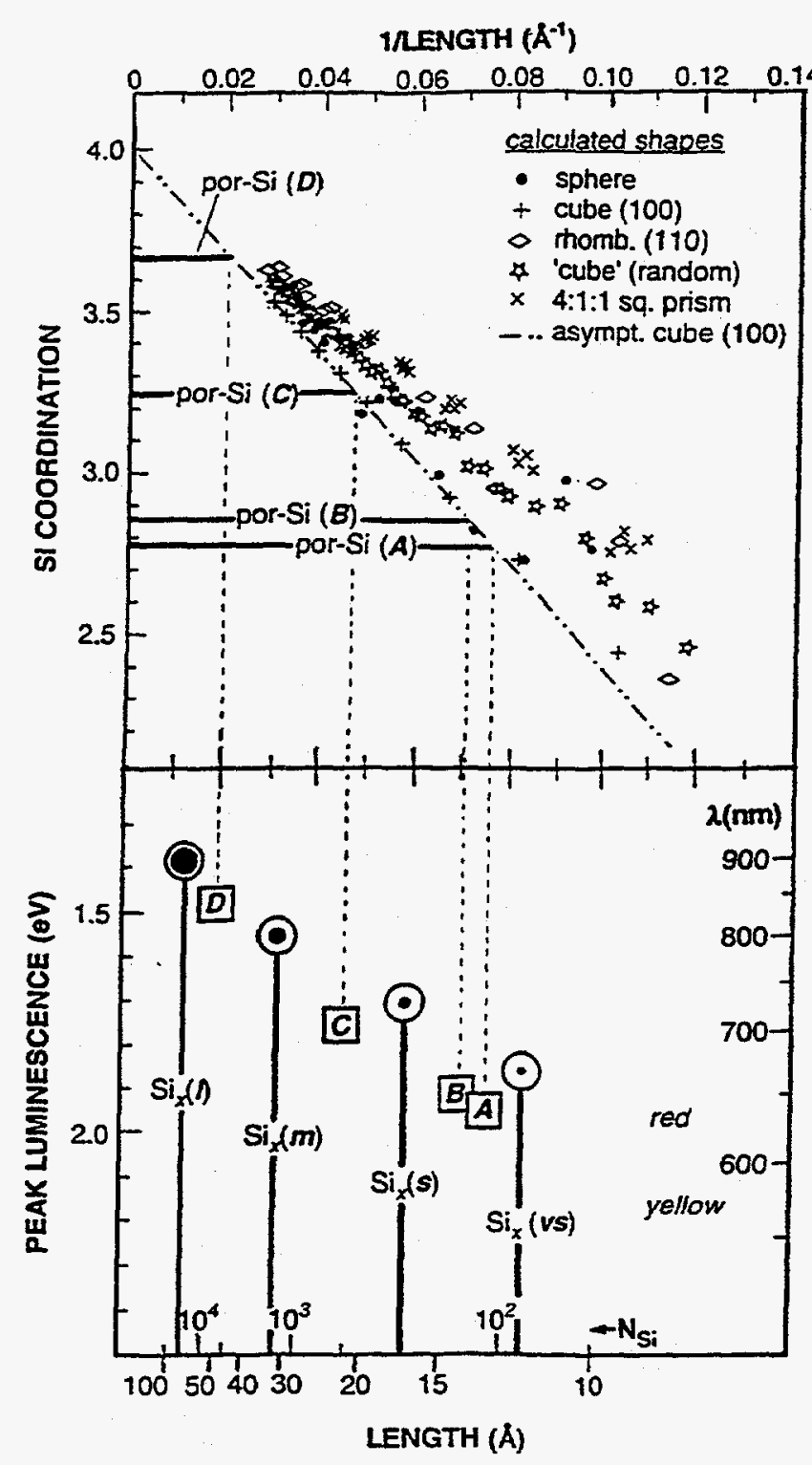

Fig. 2. Top: Correlation between average Si coordination for particles of different shapes versus their inverse characteristic length (diameter for sphere, side for cube). Average includes bulk and surface atoms, which have $<4$ first neighbors, explaining trend of lower values with decreasing particle size. Experimental Si coordinations from EXAFS data of four differently prepared por-Si samples labeled $A, B, C$, and $D$ are indicated. Bottom: Correlation between average $\mathrm{Si}$ particle size and measured peak luminescence energy. Also indicated are total number of Si atoms, $\mathrm{N}_{\mathrm{Si}}$, contained in a cubic particle of corresponding size. Different sized oxidized Si nanocrystals labeled $v s, s, m$, and $/$ are shown as circles, different por-Si structures from top figure are shown as squares.

Fig. 3. Si K-edge NEXAFS data from two oxidized Si nanocrystals, labelled medium $(m)$, and very small $(v s)$. Both spectra, normalized to unity edge jump (note different scales), are shown to be composed of three components: an $\mathrm{SiO}_{2}$ outer shell, a $\mathrm{Si}$ inner shell (here represented by a por-Si(C) sample from Fig. 1(b)), and an interfacial suboxide species $\mathrm{SiO}_{x}$ Despite very different nanocrystal sizes, the thicknesses of the oxide and suboxide shells are essentially identical.

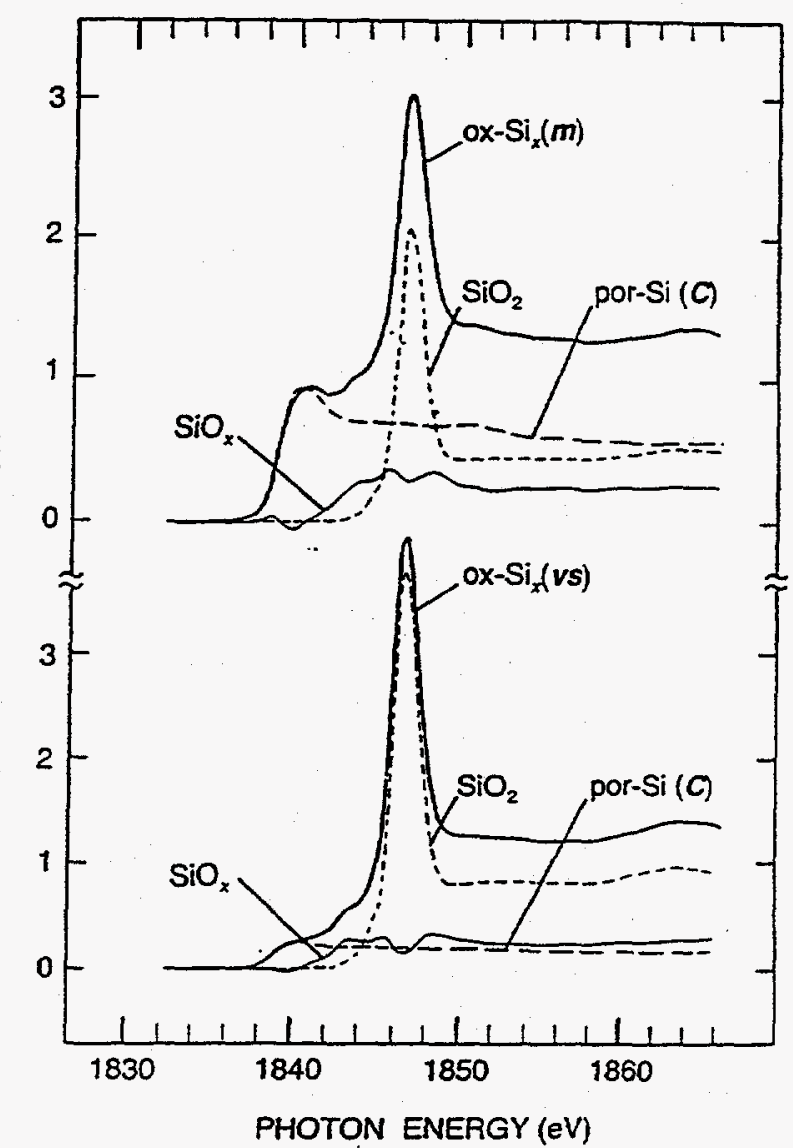


diameters are $96 \pm 19,45 \pm 7,33 \pm 6$, and $26 \pm 6 \AA$, respectively. From these values, the spherical shapes, and the relative concentrations of $\mathrm{Si}$ and $\mathrm{SiO}_{2}$ measured here, we determine the mean inner diameters of the corresponding Si cores to be $78,31,17$, and $11 \AA$. These nanocrystal sizes are plotted as circles in the bottom half of Fig. 2 versus their measured peak luminescence energies. The high quantum efficiencies of these particles ( $>25 \%$ at low temperatures [9]), the essentially constant oxide thicknesses found here (5.5 $-7.5 \AA)$ for very different diameters, and the well defined trend seen in the bottom of Fig. 2 , are all fully consistent with the $\mathrm{Si}_{x}$ cores being the optically active species in these samples. Also supporting this interpretation are recent high resolution luminescence data from these nanocrystals [10], which reveal vibronic structures directly related to Si.

Despite this strong evidence for the Si cores being the active chromophores, there have been suggestions that it is the interfacial suboxide species which is the light-emitting species. Variations in $\mathrm{SiO}_{x}$ thickness or stoichiometry with nanocrystal size are believed to account for the observed variations in luminescence peak energy. Figure 3 argues against this suggestion, showing that the suboxide component, isolated by subtracting the outer core of $\mathrm{SiO}_{2}$ and the inner core of $\mathrm{Si}$ (represented here by a por-Si sample [11], see below), is experimentally indistinguishable in the $m$ and vs nanocrystals, both in edge position, i. e., chemical composition, and relative edge jump, i. e., thickness (measured to be $1.5 \pm 0.5 \AA$ ). Identical results are also obtained for the / and s nanocrystals.

\section{NEXAFS and ir Absorption from Por-Si}

Normalized NEXAFS data from por-Si appear in Fig. 1(b), along with comparison data from $c$-Si and HF-rinsed a-Si. For clarity, we show only two of the four differently prepared samples studied here, labelied $C$ [2] and $B$ [7]. The qualitatively closer resemblance of the por-Si data to that of $\mathrm{CSi}$ rather than $\mathrm{a}-\mathrm{Si}$, and the lack of (Si-O)related absorption at $\sim 1842-1848 \mathrm{eV}$, already noted elsewhere [7], are apparent.

As in the case of the Si nanocrystals, there have been suggestions that an oxygencontaining Si species is responsible for the observed visible luminescence in por-Si. Even freshly prepared por-Si that has been minimally exposed to air contains oxygen in the form of physisorbed water trapped in the pores. This oxygen goes undetected in the Si K-edge NEXAFS data because the samples from which the $x$-ray absorption measurements are made are not exposed to exciting radiation, i. e., the oxygen remains in the form of water and does not react with the $\mathrm{Si}$. By contrast, the photoluminescence measurements necessarily involve exciting radiation, which dissociates the water and leads to formation of Si-O species. That this process of photo-induced oxidation occurs is not the issue; rather, it is whether the so-generated Si-O species is related to the observed photoluminescence.

We have investigated this point using transmission ir-absorption measurements to monitor the dependence of photo-oxidation as a function of radiation exposure. The top of Fig. 4 shows transmission data from two non-irradiated, differently prepared por-Si samples, $B$ and $C$. The bottom traces are difference spectra from those samples before and after exposure to $4 \mathrm{~min}$ of $390-\mathrm{nm}$ radiation from a high pressure Xe lamp at $\sim 2 \mathrm{~mW} / \mathrm{cm}^{2}$, conditions identical to those used in our photoluminescence measurements. In both samples there is enhanced absorption around $1100 \mathrm{~cm}^{-1}$, representative of Si-O-Si stretching frequencies, and a corresponding loss of absorption around $2100 \mathrm{~cm}^{-1}$, representative of $\mathrm{Si}-\mathrm{H}$ stretching frequencies. Expanded views of these regions in the por$\mathrm{Si}(C)$ sample are shown in Fig. 5 for different exposure times. A clear monotonic trend with exposure is observed for the $\mathrm{Si}-\mathrm{O}$ and $\mathrm{Si}-\mathrm{H}$ species (this trend saturates after $-100 \mathrm{sec}$ ). 
Significantly, however, the luminescence peak intensities and energies remain constant with radiation exposure. We therefore conclude that there is no correlation between the photo-oxidized species and the observed photoluminescence.

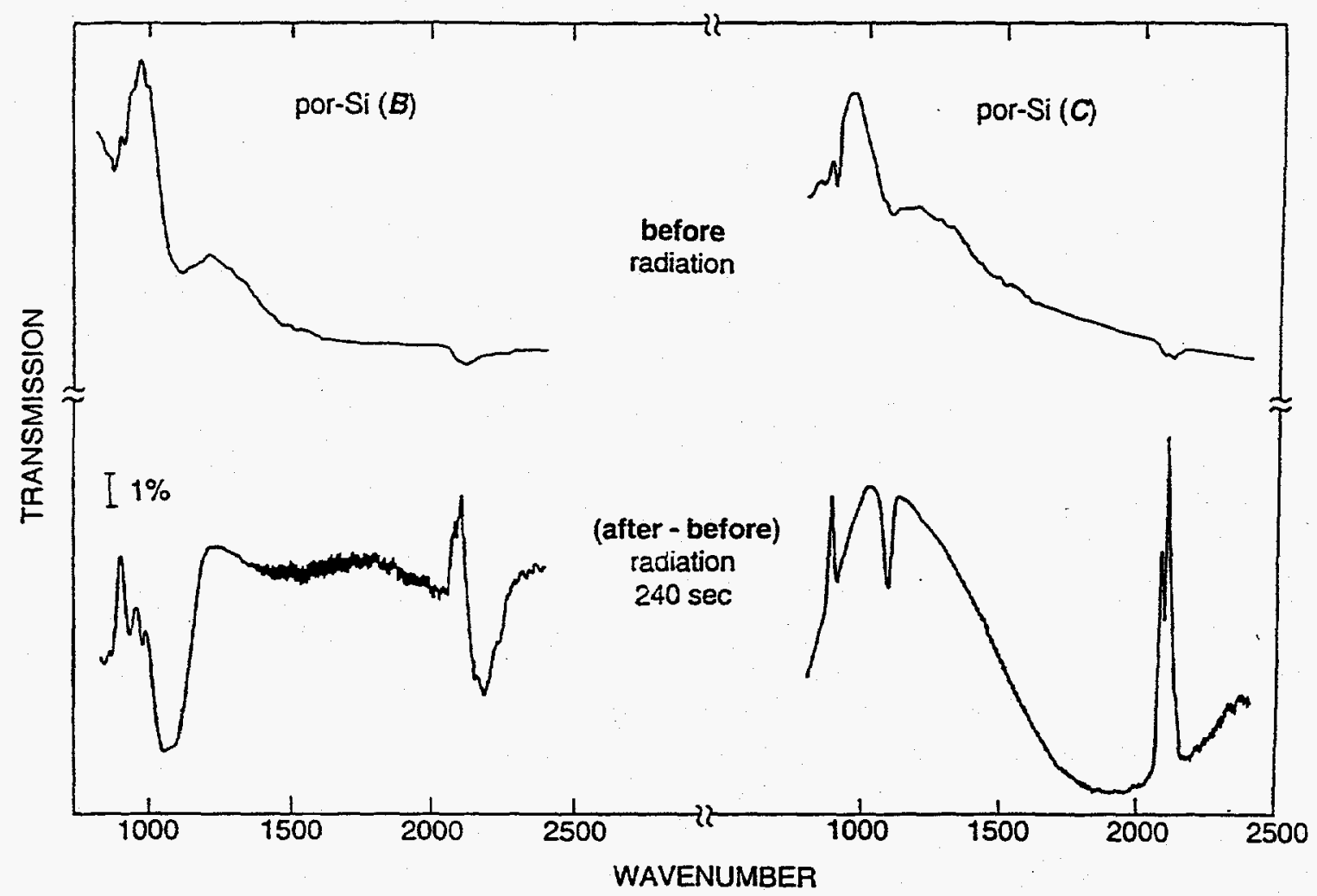

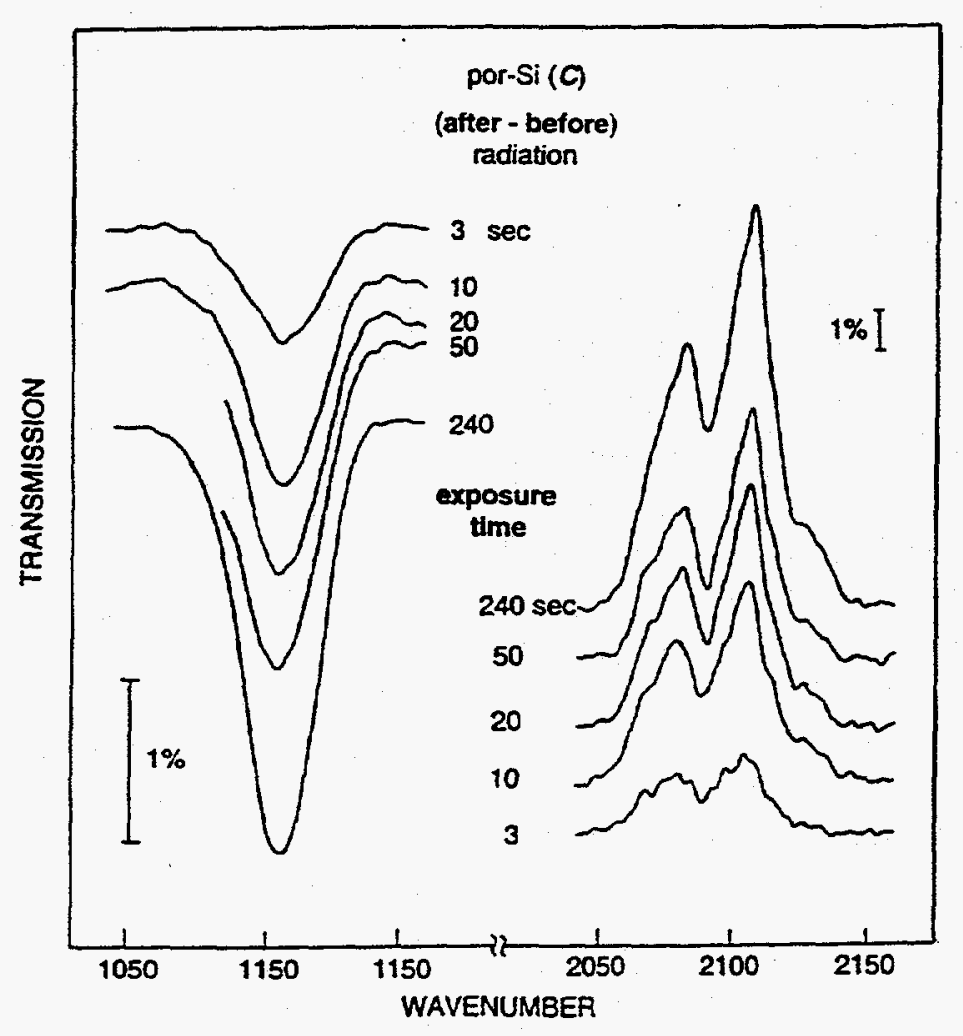

Fig. 4. Infra-red absorption data from two por-Si samples. Photo-induced oxidation due to radiation is evident in difference spectra (bottom tiaces) by appearance of Si-O species at $-1100 \mathrm{~cm}^{-1}$ with a corresponding decrease of $\mathrm{Si}-\mathrm{H}$ species at $-2100 \mathrm{~cm}^{-1}$.

Fig. 5. Infra-red absorption difference spectra from a por-Si sample showing dependence of photo-oxidized species formation and loss of Si-H species as a function of radiation exposure. These processes are unrelated to the measured luminescence intensity and peak energy (not shown), which are independent of exposure. 


\section{EXAFS from Por-Si}

While the NEXAFS data provide chemical information, i. e., electronic structure, the corresponding extended $x$-ray absorption fine structure (EXAFS) data readily provide local coordination and distance information, i. e., geometric structure [12]. In Fig. 6(a), we show the raw EXAFS data for the same samples displayed in Fig. 1(b). Locally ordered structure in the por-Si samples is seen from the second- and third-neighbor shells appearing as peaks at -3.4 and $4.1 \AA$ in the Fourier-transformed (FT) data [Fig. 6(b)]; such structure in $a-S i$ is essentially absent, of course, due to static disorder. Relative to bulk $c-\mathrm{Si}$, the apparently similar reduction of firstneighbor FT peak intensities at $-2.0 \AA$ for the por-Si and a-Si samples is better understood by filtering and back-transforming the firstneighbor shells [Fig. 6(c)]. The peak intensity of the amplitude function $A(k)$, which envelopes the oscillatory EXAFS $X(k)$, is shifted for a-Si as a result of the DebyeWaller-like static-disorder term [12]. Such exponential damping of high- $k$ scattering is unimportant for $k<4 \AA^{-1}$, so in that region $A(k)$ for the a-Si and $c$-Si samples is the same. This reflects their identical Si coordinations of 4. By contrast, $A(k)$ for the por-Si samples is unshifted relative to that for $\mathrm{c}-\mathrm{Si}$ and is smaller at all values of $k$. This is an obvious indication that the average number of first-neighbor $\mathrm{Si}$ atoms, $\eta_{\mathrm{Si}}$, in por-Si is $<4$.

The reason for the lower average $\mathrm{Si}$ coordination number and the apparent lack of other first neighbors in the FT data is that the surface $\mathrm{Si}$ atoms are also coordinated to $\mathrm{H}$ (the EXAFS backscattering amplitude of $\mathrm{H}$ is negligible). The substantially smaller values of $n_{\mathrm{Si}}$ obtained by fitting [12] the filtered por-Si data indicate large $\mathrm{H} / \mathrm{Si}$ ratios in these samples. For example, $\eta_{\mathrm{si}}=2.85 \pm 0.1$ in sample $B$, meaning $\mathrm{H} / \mathrm{Si}=1.15 \pm 0.1$, or an average $H$ content of $115 \%$. This should be compared with typical $\mathrm{H}$ concentrations of $-10 \%$ in device-quality a-Si:H [13]. Forward $\alpha$ recoil measurements [14], which determine $\mathrm{H} / \mathrm{Si}$ directly, give results for the corresponding
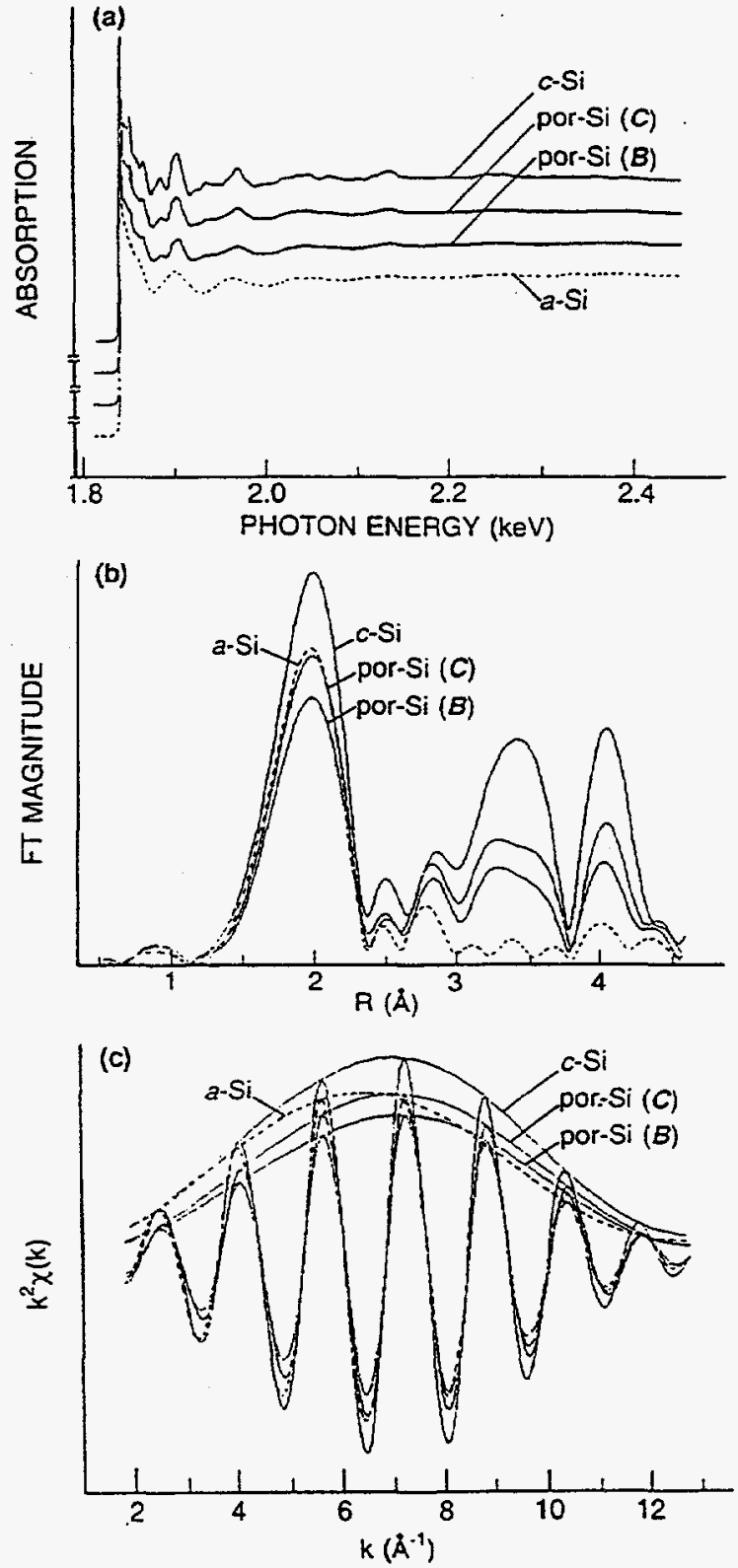

Fig. 6. (a) Raw Si K-edge EXAFS data from $c-\mathrm{Si}, a-\mathrm{Si}$, and two different por-Si samples. (b) Fourier transforms of edge-truncated, $k^{2}$-multiplied, background-subtracted data from samples in (a). 1st-, 2nd-, and 3rd-shell peaks at $\sim 2.0,3.4$, and $4.1 \AA$ are uncorrected for phase shift. Artifactual peaks at $\sim 2.5$ and $2.9 \AA$ are due to truncation. (c) Back-transformed, filtered 1st-shell data from (b). Reduced EXAFS amplitudes from por-Si samples indicate lower $\mathrm{Si}$ coordination as a result of surface coordination to $\mathrm{H}$. Measured average Si coordinations for different por-Si samples are plotted in Fig. 2. 
por-Si samples that are completely consistent with those from EXAFS. Furthermore, these integrated $\mathrm{H}$ concentrations are confirmed by transmission ir-absorption measurements [15] (the ir results also show that $\mathrm{H}$ from bulk $\mathrm{Si}$ or from possible $\mathrm{OH}$ contamination is unimportant in the $\alpha$-recoil data).

The large measured $\mathrm{H} / \mathrm{Si}$ ratios imply large surface/volume ratios for the representative Si structures, the average sizes of which are determined in the top half of Fig. 2. We first plot calculated Si coordinations for different shapes as a function of inverse characteristic dimension, or length (i.e., diameter for a sphere, side for a cube). The dotdashed line, drawn from the asymptotic limit of $\eta_{\mathrm{Si}}=4$ for infinitely large Si dimensions, is extended for (100)-faceted cubic particles of decreasing size. The 4:1:1 square-prism shapes are included to represent prolate particles. All particle surfaces (terminated with $H$ ) are ideal. To this calculated plot we then indicate the Si coordinations measured from the EXAFS amplitudes in the four different por-Si samples, labelled $A, B, C$, and $D$ (all $\eta_{\mathrm{Si}}$ values are \pm 0.1 ). Finally, the average Si particle sizes are obtained from the intersection of mean experimental Si coordinations with the asymptotic dot-dashed line. These sizes are plotted as squares in the bottom half of Fig. 2 against the peak luminescence energies measured in the corresponding samples. A correlation is obvious.

\section{DISCUSSION}

Before discussing the trend at the bottom of Fig. 2, we point out that the particle sizes represented by the squares are actually upper limits. The quoted particle sizes are obtained from the (100)-cubic asymptote, so any other assumed shape(s) would clearly lead to smaller sizes. Furthermore, TEM micrographs of the por-Si samples show them to contain $c-S i$, often in the form of columns, whose dimensions are $\geq 3 \mathrm{~nm}$ and whose amounts vary between $10-30 \%$ depending on the type of sample studied, e. g., $B$ versus $C$. The remainder of the samples consists of $\mathrm{Si}$ structures too small to characterize with TEM. Since EXAFS measurements average over all the Si structures, including the contributions from $c-S i$, the reduced values quoted for $\eta_{\mathrm{si}}-$ and thus the mass-weighted average particle sizes - would be even smaller had these larger $c$-Si contributions not been included. It is significant to note that subtracting the $c$-Si contributions from the por-Si data still leaves evidence for local crystallinity, i. e., the 2nd-and 3rd-neighbor shells in the FT data are not removed.

The bottom of Fig. 2 displays the important and surprising result that the relationship between peak luminescence energy and average particle size for the por-Si samples is nearly identical to that for the Si nanocrystals. Indeed, excluding the unetched $c$-Si contributions to our quoted $n_{\mathrm{Si}}$ values make the two trends virtually indistinguishable. The implication is clear: since the luminescence spectrum from a given Si particle is directly correlated with its size, the luminescence spectrum from a given por-Si sample is also associated with a characteristic Si dimension. Thus, for example, $720-\mathrm{nm}$ peak luminescence from $\mathrm{Si}$ is representative of a structure whose average size is $\sim 20 \AA$, regardless of how the sample was prepared.

The correlation in Fig. 2 also provides information about the shape of the Si species responsible for the luminescence in the visible region $<700 \mathrm{~nm}(>1.75 \mathrm{eV})$. The columnar, extended-wire-like structures observed with TEM in por-Si samples $A$ and $B$ are all $>20 \AA$, i. e., too large to account for our EXAFS results. If extended wire shapes were responsible for the luminescence in these samples, they would have to be of extremely small 
dimensions, $<10 \AA$. This implies, therefore, that the optically active structures are generally not extended wires, but particles. We infer that these particles are predominantly (100)faceted based on surface sensitive reflection ir-absorption measurements [16] from the por-Si $A$ and $B$ samples, which indicate $\mathrm{H}$-Si frequencies representative of $\mathrm{SiH}_{2}$ species [(110) or (111) surfaces would exhibit mainly $\mathrm{SiH}$ frequencies]. More general conclusions cannot be extended to samples luminescing in the near- and far-infrared region, i. e., $>700$ $\mathrm{nm}$, because the distinction between wires and particles [see Fig. 2] is less significant.

The average sizes of the Si structures responsible for the visible luminescence in por-Si are small, well below easy detection with TEM or diffraction. For example, Fig. 2 indicates that 2-eV luminescence is associated with particles of dimensions typically less than $13 \AA$ (recall that the values shown in squares are upper limits). This is considerably smaller than any size previously associated with such luminescence. A 13-Å cube contains a total number of Si atoms $\mathrm{N}_{\mathrm{Si}} \approx 110(\approx 60$ for a sphere [17], see Fig. 2) and a calculated band gap of $\sim 3.8 \mathrm{eV}[18]$, whereas a $26-\AA$ cube has corresponding numbers of -900 and $2.2 \mathrm{eV}$. Factors of two in size are therefore very important.

Basic questions about visible photoluminescence from por-Si still remain. Among these is a reliable calculation of the size of the coulombic interaction between electrons and holes localized on Si particle. A complete description of the visible-luminescence mechanism in por-Si will ultimately need to address this and other issues, along with the fact that the optically active Si species in this material has dimensions as small as those found here.

\section{ACKNOWLEDGEMENTS}

S. Schuppler is grateful to the Alexander-von-Humboldt Foundation for financial support. The EXAFS experiments were performed at the NSLS, Brookhaven National Laboratory, which is supported by the DOE, Division of Materials Sciences and Division of Chemical Sciences. The TEM measurements, performed at the National Center for Electron Microscopy, LBL, are supported by the Director, Office of Basic Energy Sciences, DOE, under contract number DEAC03-76SF00098.

\section{REFERENCES}

a) Present address: Department of Applied Physics, Stanford University, Palo Alto, CA 90210

b) Present address: Department of Materials Science, University of Illinois, UrbanaChampaign, IL 61801.

1. For example, see (a) Mater. Res. Soc. Proc. 256 (1992); (b) ibid. 283 (1993); (c) ibid. 298 (1993).

2. L. T. Canham, Appl. Phys. Lett. 57, 1046 (1990)

3. V. Lehmann and U. Gösele, Appl. Phys. Lett. 58, 856 (1991).

4. S. Schuppler, S. L. Friedman, M. A. Marcus, D. L. Adler, Y.-H. Xie, F. M. Ross, T. D. Harris, W. L. Brown, Y. J. Chabal, L. E. Brus, and P. H. Citrin, Phys. Rev. Lett. 72, 2648 (1994).

5. A. A. MacDowell, T. Hashizume, and P. H. Citrin, Rev. Sci. Instr. 60, 1901 (1989). 
6. Preparations of the por-Si samples studied here (referred to as $A, B, C$, and $D$ ) follow those in Refs. 2, 7, and 8, namely, $C$ [2]: p-type Si(100), $>50 \Omega-\mathrm{cm}, 20 \% \mathrm{HF}$ in alcohol, $20 \mathrm{~mA} / \mathrm{cm}^{2}$ for $5 \mathrm{~min} ; A$ [8]: same as $C$, but etched $60 \mathrm{~min} ; B[7]$ : $p$-type $\mathrm{Si}(100),>50 \Omega$ $\mathrm{cm}, 15 \% \mathrm{HF}$ in alcohol, $25 \mathrm{~mA} / \mathrm{cm}^{2}$ for $12 \mathrm{~min} ; D$ [8]: p-type Si(100), 0.5-0.8 $\Omega$-cm, $40 \%$ $\mathrm{HF}$ in alcohol, $50 \mathrm{~mA} / \mathrm{cm}^{2}$ for $80 \mathrm{sec}$, soaked $2 \mathrm{hr}$ unetched in same solution.

7. S. L. Friedman, M. A. Marcus, D. L. Adler, Y.-H. Xie, T. D. Harris, and P. H. Citrin, Appl. Phys. Lett. 62, 1934 (1993).

8. Y. H. Xie, M. S. Hybertsen, W. L. Wilson, S. A. Ipri, G. E. Carver, W. L. Brown, E. Dons, B. E. Weir, A. R.Kortan, G. P. Watson, and A. J. Liddle, Phys. Rev. B 49, 5386 (1994); Y.-H. Xie (unpublished).

9. Oxidized Si nanocrystals were made by homogeneous nucleation in high-pressure $\mathrm{He}$ at $1000^{\circ} \mathrm{C}$ from thermal decomposition of disilane with subsequent oxidation in $\mathrm{O}_{2}$ at $1000^{\circ} \mathrm{C}$ for $\sim 30 \mathrm{msec}$. See K. A. Littau, P. J. Szajowski, A. J. Muller, A. R. Kortan, and L. E. Brus, J. Phys. Chem. 97, 122 (1993); W. L. Wilson, P. J. Szajowski, and L. E. Brus, Science 262, 1242 (1993); P. J. Szajowski and L. E. Brus (unpublished).

10. L. E. Brus, P. F. Szajowski, W. L. Wilson, T. D. Harris, S. Schuppler, and P. H. Citrin, J. Amer. Chem. Soc. (to be published).

11. This por-Si sample more closely approximates the Si core because it contains less long- range order than $c$-Si. The results are completely unaffected by this choice because only difference spectra are being compared.

12. P. A. Lee, P. H. Citrin, P. Eisenberger, and B. M. Kincaid, Rev. Mod. Phys. 53, 769 (1981).

13. R. A. Street, Hydrogenated Amorphous Silicon (Cambridge University Press, Cambridge, 1991).

14. B. L. Cohen, C. L. Fink, and J. H. Degnan, J. Appl. Phys. 43, 19 (1972).

15. The volumes used for integrating the measured $\mathrm{SiH}_{x}$ concentrations in the por-Si samples were obtained from TEM.

16. Surface sensitivity of $\langle 1 \mu \mathrm{m}$ was obtained using a grazing internal incidence angle in a Ge plate positioned next to the por-Si samples.

17. For a Si cube of side $L, N_{S i}=8 L^{3} / a_{0}^{3}$; for a sphere of diameter $L, N_{S i}{ }^{s p h}=(\pi / 6) N_{S i}{ }^{\text {cube }}$.

18. See, e. g., C. Delerue, G. Allan, and M. Lannoo, Phys. Rev. B 48, 11024 (1993), and references therein. 